\title{
Social Science Research and Everyday Human Life
}

\author{
Prakash Upadhyay, PhD
}

\begin{abstract}
Pedestaled on qualitative secondary data congregated from secondary sources, the major objective of this paper is to analyze relation between social science research and daily human life especially with problem solving and decision making activities. The key argument of this paper is that social science research attempts to find social patterns of regularity in social life that usually deals with social group. Yet, in social science there are no social laws parallel to natural science laws. A law in social science is a universal generalization about a class of facts. There are diverse schools of thought embedded with key perspectives in social research. Social researchers inquire and wonder on diverse issues and matters in everyday human life. By means of sense and inquisi tive knowledge background they explore the raison d'être behind any issue. And it is the systematic research probing the reasons behind the issues tremendously germane in daily life. In our daily life we put up with copious tribulations, research knowledge and the solutions of the problems emerging from researches prove handy and practical for the society and the people. This study deduced that, improving the quality of every day social life is a significant goal since the pools of ideas configures much philosophy and much telling about society, by social scientists and by others. Hence, in having a comprehension of debates and the applicability of different methods of research and the implications of research findings on society, advanced researches and more inquiring and confident researchers will be more productive.
\end{abstract}

Keywords: Axioms, intellectual application, positivism, scientific cloak, substantive hypothesis

\section{Background}

Research is the rummage around for knowledge, any systematic investigation, with an open mind, to establish fresh facts, usually by using a scientific method, an investigation or analytical process in which some facts are brought forward to the populace such facts that already exits but the people have no any awareness about them. As a way to the intellectual application of methods, it is the investigation of matter used for discovering, interpreting and development of methods and systems for advancement in human knowledge (Upadhyay, 2011).

In the pursuit of knowledge embed with human lives, society and other quarters research is principally a unique process of movement. Kothari (2006) says research is the search for knowledge through objective and systematic method of finding solution to a problem. It is essentially a technique of critically thinking by defining and redefining problems, formulating hypothesis or suggested solution, collecting, organizing and evaluating data, making deductions and making conclusions to resolve whether they fit the formulated hypothesis. If a 
research focuses its attention on objectives and objectivity, it is called scientific. Nevertheless, the research ought to be unbiased, liberated of prejudice, based on full evidence and inclusive in itself. In scientific research it is prerequisite that there should be the use of both quantitative and qualitative techniques and use of direct and cogent language. The methodology for the task of undertaking a research should be reliable. In another condition, if another researcher (s) carries on the same research, the result should be similar to some extent.

Correspondingly, research methods are a fundamental part of the social sciences providing a means through which intellectual development and progress is enhanced. Indeed, the status as 'sciences' is often justified by attaching to the technical features of research methods, while the very term 'science' carries with it ideas of areas of study which are easy to get to only to those who have undergone an wide-ranging training process in order to understand their internal workings. Theory, methods and methodology are all part of the issues and processes that surround and inform the discipline. These differences, however, constantly lead to disputes, as well as confusions, over the nature of research and the methods employed in following its aims and the use in daily life.

\section{Problem and Objectives}

Everyday life and research is entrenched and researches can be accomplished in everyday life situation of human being. It also denotes that researches are very much rooted in daily life of the people. But, as there are diverse schools of thought embedded with key perspectives in social research, social researchers inquire and wonder on diverse issues and matters in everyday human life. Given this significance, the key objective of this paper is to analyze relation between social science research and daily human life. The main focus is on how researches in every day life are embedded with problem solving and decision making activities.

\section{Data and Methods}

As this perusal is based on secondary review, no primary field data has been used here. The paper is solely based on qualitative secondary data congregated from different secondary sources viz. Library books, journals, booklets and internet sources.

\section{Research and Every Day Life}

In our everyday life we inquire, wonder and ponder on diverse issues and matters. Using our sense and inquisitive knowledge background we explore the reasons behind any issue. And it is a research as we are searching the reasons behind the issues. In fact, researches are extremely relevant in day to day life. In our daily life we face numerous problems and the research knowledge and the solutions of the problems emerging from researches are becoming very handy and practical. Researches are and can be performed in diverse sectors related especially with issues and problems which are embedded with our routine daily verve, cognitive, social-cultural life and involvements etc.

Focusing on diverse social and cultural ills (problems) a narrow attitude to social research 
practice supports the idea that theory, ethics, values and methods of social research are distinct topics and those researchers, despite living and participating in the societies that they study, are somehow distinct from the social world which is the object of their investigations. Nevertheless, the findings of their studies may be extremely helpful to the society and for the people living there. In having an understanding of debates and the applicability of different methods of research and the implications of research findings on society, improved research and more inquiring and confident researchers will be more productive. Issues and methods cannot be simply separated and hence researchers will produce more systematic understandings of the social world by being aware of problems, their implications for research and applications of research findings on society and day to day life.

Researches and everyday life are embedded and researches can be accomplished in everyday life situation. It means researches are also very much embedded with everyday life of the commoners. There is nothing ordinary about the ordinary, anthropologists' claim. That is, if by ordinary we mean usual or normal nevertheless, members of every culture believe that their way of doing things is normal. If we look at the world from the viewpoint of people living in a particular society, we can without obscurity point to the ordinary pleasures, rituals and taboos that the culture associates with its food. French anthropologist Claude Levi-Strauss in The Raw and the Cooked (1964) studied what people ate and how different cultures prepare their food, through their myths, in order to identify the underlying structures of human thought. In every culture, he tells us, people put the food they eat into three broadly defined categories, two natural (raw and rotten), one mediated by cultural intervention (cooked).

To help visualize the relationship, Levi-Strauss imagines a "culinary (cooking) triangle". Strauss says that people spend most of their time in almost every culture turning nature into culture, the raw into the cooked. However even when they value culture over nature, they frequently break their own rules, defying their own categories. Cultures may favor cooked food over raw and still prize delicacies that are uncooked. A Nepali meal, for example, could easily begin with a glass of cold water (Raw) for washing hands, followed by Dal, Bhat, Dhido, Gundruk cooked for hours, and end with a selection of (rotten) curd or milk and betel nuts, tobacco etc. Still cultures make choices and are favored by the people belonging to that culture and hence, researches can be made on such trivial issue which reserves a major role in daily life of the people living in different cultures and societies.

Squabbling on research and everyday life interrelationship Young (2002) is concerned more with diverse issues. She squabble that one main question for concern is in the main, with what do social researches concern themselves? On what general assumptions do they base their researches? And briefly stated: Research for what? The answer is .... researches for solving the problems of everyday life. Working out the practical tribulations, grievances and everyday trivial issues is the answer. From the knotty topics to the trivial issues, for example, of eating and sleeping may be the researchable topics. For example Mary Douglas (1966) in her highly study of Kashrut, tried to make order out of the dietary practices of Jews, focusing, in particular, on two central concepts in Leviticus: tehvel and kadosh. To study everyday life would be a completely strange undertaking, unable even to grasp anything of its object, if the study is not specific for the purpose of analyzing and transforming everyday life of the people. 
Research scholars are excessively inclined to exclude from everyday life things that happen to them every day, and to transfer them to separate and supposedly superior spheres. In this way habit in all its forms - beginning with the habit of handling a few professional concepts (concepts produced by the division of labor) — masks reality behind privileged conventions. The reality of an observable entity designated by the term everyday life stands a good chance of remaining hypothetical for many people. Indeed, the most outstanding feature of research and everyday life is not the fact that it has not yet discovered anything, but the reality that the very existence of everyday life is very crucial for the comprehension of social-cultural processes and peoples behavior in daily life.

\section{Scientific Research: How a Research Becomes Scientific?}

Science is a systematic knowledge based on rigorous experimentation and field work. It is a knowledge attained through study or practice, or knowledge covering general truths of the operation of general laws. Based on the system of acquiring knowledge science uses observation and experimentation to describe and explain natural phenomena. The purpose of science is to produce useful models of reality. Nearly all scientific investigations use some form of the scientific method.

The fields of science are usually organized along two major lines, Natural sciences dealing with the study of the natural world, and Social sciences with the systematic study of human behavior and society. Social sciences are also based on rigorous scientific research. Kerlinger (1996) has alleged that scientific research is a systematic, controlled, empirical and critical investigation of hypothesis propositions about the presumed relationships among natural phenomenon. In this context Scientificness does not essentially mean science but it refers to the manner of doing research activity that is step-by-step, logical, organized and rigorous method to identify problems, data collection, and analysis and draw valid conclusion about the data. Scientificness is not based in perception and speculations or guesses.

The standpoint that is universal to all search methods and techniques is the adoption of scientific method committed to objectivity, utilizing relevant concepts, describing methodology and formulating theories. The principal goal of research is the search of truth and knowledge determined by logical considerations. Typical qualities of scientific research are that it depends on empirical evidence/data and is committed to objectivity. Devotion to ethical objectivity is the feature of scientific research. It describes methodology used and aims at clarifying concepts and formulating theories.

Scientific research determines recognized problems, paradoxes, and/or irregularities irresolvable on the basis of earlier theories. It create new set of scientific problems for scientists to work on and suggest a paradigm or problem solving model to facilitate resolve new problems and provide definitions of concepts or operations which help other scientists solve problems. Scientific research achieves two vital functions, the development of theory and testing the substantive hypothesis that are deduced from the theory. A research will be called scientific in its spirit if it is well organized and every step is linked to each other. Moreover, scientific spirit implies that truth has to be based on observable facts and evidence. It should 
be objectively analyzed. If a research focuses its attention on objectives and objectivity, it is called scientific. Yet, the research ought to be free from prejudice, based on evidences and complete in itself.

There should be the use of both quantitative and qualitative techniques and use of direct and simple language. The methodology followed to undertake a research ought to be reliable. In another condition, if another researcher (s) carries on the same research, the result should be similar to some extent.

Scientific research is a method for the organized investigation of issues based on certain explanation (layout). Joshi (2002) presents a common layout of scientific research:

\section{Figure 1: The Scientific Research Process}

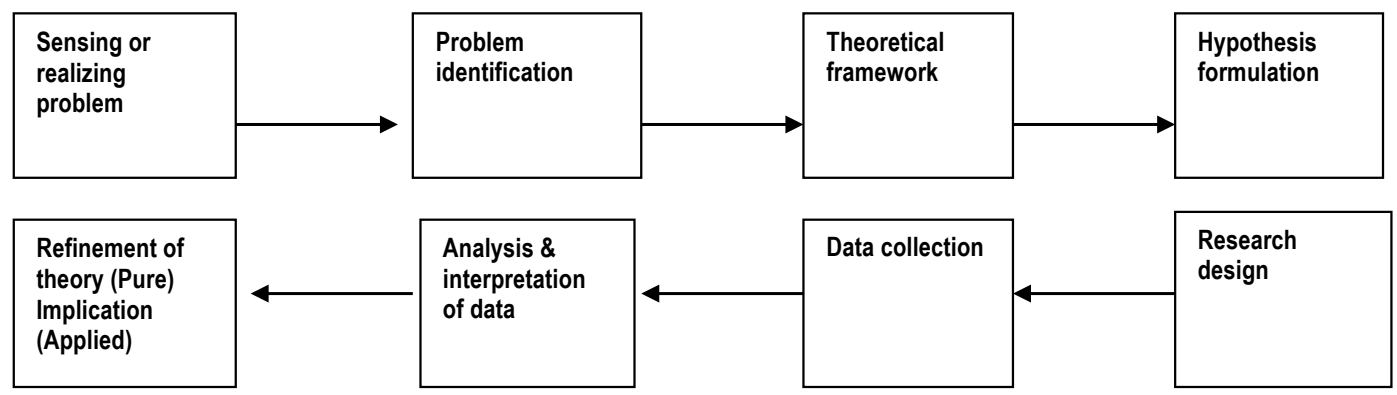

Source: Joshi, 2002

Scientific research is based on a system of ideas, a body of statements. Only logical consistency and evidence can serve as a check, a way of evaluating the accuracy of ideas. The purpose is to find order in the chaos of natural phenomena and it attempts to represent knowledge as simply and accurately as possible with natural laws.

\section{Schools of Thought in Social Research}

Logic and empirical observations are the part of social research. Social research is concerned with the relations between ideas and evidence. Ideas help social researchers make sense of evidence, and researchers use evidence to extend, revise and experiment ideas. Social research tries to create or validate theories through data collection and analysis, and its goal is exploration, description and explanation. It ought to be noted that social research should never lead or be mistaken with philosophy or belief. It is a fact that social research aims to find social patterns of regularity in social life and usually deals with social groups. However, in social science there are no social laws parallel to natural science laws. A law in social science is a universal generalization about a class of facts. A fact is an observed phenomenon, and observation means it has been seen, heard or otherwise experienced by researcher. A theory is a systematic explanation for the observations that relate to a particular aspect of social life. Concepts are the essential building blocks of theory and are abstract elements representing classes of phenomena. Likewise, axioms or postulates are fundamental assertions 
assumed to be true and the propositions are conclusions drawn about the relationships among concepts, based on analysis of axioms. Similarly, hypotheses are tentative generalizations or the specified expectations about empirical reality which are derived from propositions. Social research involves testing the hypotheses to see if they are true.

Related to schools of thought in social research the key perspectives in social research are diverse. Positivism's approach is based on scientific notion with the idea that social science ought to reflect the aims and methods of natural science however the assessment of different methods clarifies that social researches seems less objective compared to natural sciences. Realism's notion is that knowledge the people have of their social world influence their behavior, and feminist criticisms of the foundations and aims of science as being malecentered and hierarchical, finishing on a critique of research practice as ethnocentric and racist reveal the diversities in the schools of thought in social research. The multiple discussions themselves are complex, but no less significant for that. However, all these perspectives do not simply dictate the nature of research itself, or how it is hold out; even though the issues will inform how the aims, methods and process of social research are measured.

\section{Positivism}

The chances for deriving different types of meanings from the same sort of social situations have contributed to generate debates on the positivists and the interpretative line of discussions of human relations (Pandey, 2008). French mathematician, Auguste Comte first described the epistemological perspective of positivism. Observing the circular dependence of theory and observation in science, and classifying the sciences, Comte may be regarded as the first philosopher of science in the modern sense of the term. He proposed that social ills could be remedied through sociological positivism, an epistemological approach. This approach asserts that we can, to some extent, control our own future rather than have it controlled, like the molecules, by a change in our environment. Human can 'act on', as well as behave in 'reaction to', their social environments. Some experiments in the natural sciences take the form of changing the environment and considering how people react to it. The problem here is one of controlling the inputs into experimental situations in order to see what changes cause particular alterations in behavior. There are problems with this approach; hence the idea of 'quasi-experimentation' is an approximation towards this model of research. In some approaches to evaluation research, for example, a group is chosen who will be subject to the program which is to be assessed. This treatment group will then be compared to those who have not been subject to the program. However, it is necessary to raise questions- is social life really like that? Are experiments in a laboratory artificial and so doing not reflect the problems, decisions and inconsistencies involved in society?

French sociologist Emile Durkheim rejected much of the detail of Comte's philosophy, he retained and refined its method, maintaining that the social sciences are a logical continuation of the natural ones into the realm of human activity, and insisting that they may retain the same objectivity, rationalism, and approach to causality. Durkheim argued: "our main goal is to extend scientific rationalism to human conduct... What has been called our positivism is but a consequence of this rationalism." 
For the researchers leaning toward positivism, the social scientist ought to study social phenomena 'in the same state of mind as the physicist, chemist or physiologist when probing into a still unexplored region of the scientific domain. Objectivity is then defined by being the same as that of natural science and social life may be explained in the same way as natural phenomena. This tradition may therefore be characterized in terms of the prediction and explanation of the behavior of phenomena and the pursuit of objectivity, which is defined as the researcher's 'detachment' from the topic under investigation. The results of research using this method of investigation are then said to produce a set of 'true', precise and wide-ranging 'laws' (known as covering laws) of human behavior. We would then be able to generalize from our observations on social phenomena to make statements about the behavior of the population as a whole. In broad-spectrum Positivism explains human behavior in terms of cause and effect with empiricism as the feature. If the aim of positivism is to collect and assemble data on the social world from which we can generalize and explain human behavior through the use of theories, then it shares with empiricism the belief that there are 'facts' which we can gather on the social world, independently of how people interpret them. Researchers simply need to refine their instruments of data collection in order that they are neutral recording instruments much as the ruler measures distance and the clock, time. The fundamental difference between empiricism and positivism, however, lies in the realm of theory. Data within positivism is theory-driven and designed to test the accuracy of the theory. Empiricism, on the other hand, is a method of research which has not referred explicitly to the theory guiding its data collection procedures. It is thus characterized 'by the catchphrase "the facts speak for themselves.

Prominent sociologist Giddens (1974) noted that the positive attitude in sociology attempts to make deliberations on the basis of the following three types of connected suppositions. They include:

- The methodological procedures of natural sciences may be directly adapted to sociology. Human subjectivity does not create any barrier to treat social conduct as objects in the same way as are treated in the natural events.

- Sociological analysis can help to formulate laws or law-like generalizations of the same kind as those which have been established in relation to natural reality.

- The findings of sociological analysis do not carry any logically given implications for practical policy or for the pursuit of values.

The positivist thinkers do not pay any attention to take into account the role played by humans as subjects in the process of formation of their social situations. Their arguments are based under the conception that social relations are given like the natural events. The role of social scientists is to describe the objective content observed in these relations. The sociologist, even if it is a human subject, holds a neutral stand in the process of collection and interpretation of data available in empirical field for the understanding of observed reality. It remains as a neutral observer and a technical person unaffected by any personal values and moral prescriptions.

Those who consider sociology as an interpretive science raise questions on positivist assumptions. They hold the perception that humans are the conscious actors of society. Their 
conducts are guided by some interests or objectives. They proceed in these behaviors through conscious evaluation of their situations. They derive through their experience the standards used for the evaluation of those situations. Any changes in those situations bring changes in behaviors of people and in the standards used by them to evaluate these changed situations. Social relations are, therefore, neither given nor are the static phenomena. They are created by interest based interactions of people and are changed with changes in those interests and related interaction. An assignment of natural attributes to the subjectively created social situation has made many implications to the type of knowledge generated by adopting the positivist brand of thought and methodological principles.

\section{Realism}

Realism has an extended past may be linked with the works of Karl Marx and Sigmund Freud. Under this thought the tasks of researchers are to organize one's concepts so as to grasp its essential features successfully. If researchers simply content themselves with studying everyday social life, such as conversations and interactions between people, this will distract them from an investigation of the underlying mechanisms which make those possible in the first instance. The task of researchers within this tradition is to uncover the structures of social relations in order to understand why we then have the policies and practices that we do.

According to the concept of Realism the knowledge people have of their social world affects their behavior and, unlike the propositions of positivism and empiricism, the social world does not simply 'exist' independently of this knowledge. Given this, causes are not simply determining of actions, but must be seen as 'tendencies' that produce particular effects. Yet people's knowledge may be partial or incomplete. The task of social research, therefore, is not simply to collect observations on the social world, but to explain these within theoretical frameworks which examine the underlying mechanisms which inform people's actions and prevent their choices from reaching final result.

Realism utilizes a different definition of science to positivism. In particular, a realist conception of social science would not essentially take for granted that we can 'know' the world out there independently of the ways in which we illustrate it. Access to these different layers of reality is the task of a realist research program and revealing to the notice of peoples how they affect their actions in a situation of dialogue and cooperation. This is Realist philosophy, in contrast to other approaches to assessment.

Positivism does not pay a great deal attention to the detail of people's inner mental states. However Realism, on the other hand, may refer to people's consciousness in so far as it reflects the conditions under which they live, how structures are reproduced and their desires and needs are frustrated. Realist focuses on the meanings that people give to their environment, not the environment itself.

\section{Idealism}

Idealism school of thought emphasizes human creation of social world through the realm of ideas, rather than our being simply conditioned or created by it. They would argue that our 
actions are not governed by cause and effect, as in the case of molecules in a test tube, but by the rules which we use to interpret the world. As natural science deals with matter which is not 'conscious', researchers of this thought argue that its methods cannot deal with social life and should therefore be discarded from its study. To speak of cause and effect is not applicable to researching social life for people contemplate, interpret and act within their environments. For these reasons, the methods of the social sciences are basically different from, but not inferior to, the natural sciences. It is the world of ideas in which we are interested as social researchers. Such a viewpoint suggests that human activity is not behavior (an adaption to material conditions), but an expression of meaning that humans give (via language) to their conduct.

Rules exist in social action through which we produce society and understand and recognize each other. Rules, of course, are often broken and also subject to different interpretations. For that reason, we cannot predict human behavior but people still act as if they were following rules and this makes their actions intelligible. People are constantly engaged in the process of interpretation and it is this which we should seek to understand. In other words, researchers should concentrate upon how people produce social life. Social life can be understood only through an examination of people's selection and interpretation of events and actions. There are also many scholars who have attempted to fuse some aspects of major perspectives. For example Pierre Bourdieu's (1992) work has been determined by empirical interests and so has developed a series of 'tools' for researching and thinking about the social world. Giddens (1974) has argued that our everyday actions are meaningful to us, but they also reproduce structures which both facilitate and restrain human actions.

\section{Feminism}

Feminist claim that more women were prevented from understanding social relations because men, either purposely or any other reason, stood in the way of their aspirations. The ideas and practices of men reflected a bias which defines society and science in terms of particular male values. Not only would the aims of research then be incomplete, but also its results would be a distortion of the social world. Science reflects certain views of women by providing a scientific cloak but which is no more than prejudice. The arguments that male values are dominant form the starting-point of feminist criticisms of science. Women and their fundamental contributions to social and cultural life have been marginalized and this is reflected in research practice. The norms of science bring about and disguise the symbol of the superiority of men over women and imitate a desire to control the social and natural worlds. When the roles of women are considered in social life, they are characterized as submissive and emotional. This assumption has long been confronted by groups of women. Feminists argue that women's position within society is not a natural phenomenon, but a social, political and economic product which is reflected and perpetuated by the bias of science. According to feminist critiques, economics, politics, sociology, social policy and other social sciences have, like the natural sciences, perpetuated this myth. For example, in the earlier studies on the family and work, before feminist research had some impact on dominant practices, women were 'wives', 'mothers' or 'housewives', but not people in their own right. Feminists believe that social research often focuses selectively in the public realm: 
it is men who paint pictures, men who think about the world, men who make money and men who shape women's destiny. In research on the world of work, researchers function according to definitions which remain unchallenged and so it is clear that sex discrimination continue in the workforce. Feminisms, while correcting the class-based accounts of research, have faced similar criticisms as research in general, for its neglect of the issue of race. Researchers have documented the ways in which sexual and racial stereotypes cut across each other.

\section{Postmodernism}

Postmodernism is ideological movement that explains, and analyzes different sectors of the present global society viz. economy, society, culture, art, language, literature, ideals etc from a new angle. It stood as critical to modernist approach to society which presents integrated ideas of the future society as it put emphasis on human values. As a movement, postmodernism deals with the changes of the society involving a revolution in thinking, behavior, art, culture, and architecture. A philosophy based on the notion of broad spectrum criticism of modern society and all fields of human achievements, the basic concepts and premises of post-modernism are diverse and complex. Postmodernism is often described as an orientation within the philosophy of science, which denies the possibility of acquiring factual knowledge about the world. It believes that what we know about society is our own construction, which we must deconstruct. The great narratives (development, science, freedom, romanticism, truth) are heroic myths that give legitimacy to the existing social order. In social sciences, such thoughts have in particular influenced our understanding of reflexivity and our critique of Western hegemonic ideas. Postmodernism has at least two meanings:

- As a descriptive label for a specific historical era, characterized by fragmentation of dominant Western myths and collage-like assemblages of meaning (in this sense, postmodernism is related to such terms as "late capitalism" and "post-industrial society"), and

- As a term for the above-mentioned academic and artistic schools, which consider fragmentation and collage (random collection) as esthetic or intellectual ideals. It is hence possible to agree that a postmodern historical era has arrived, without subscribing to postmodernism as a model of academic inquiry.

It has been said that the very reality of the emergence of a post modernist world has inevitably set up a new agenda for the social sciences (the agenda of postmodernism and its theoretical stance within and towards this world) since the existing one and its whole set of concepts, assumptions, aspirations, method are no longer relevant. Thus, its foundations lie in the investigation of and programmatic attempt to control and direct the highly modern world. Postmodernism shares with feminist relativism the faith that knowledge is both local and contingent and there are no standards beyond particular contexts through which we may judge its truth or falsity. Therefore, it is important to note that one does not necessarily have to become a postmodernist in order to accept some of its insights regarding the changing times through which we are living. Postmodernism is often used as a convenient label around which to base a series of observations on contemporary issues (Bauman 1997). Postmodernists are anti-foundationalist. Whether talking about implosions of meaning with the consequence 
that the world becomes devoid of any meaning, or of the computer age and the severing of the link between knowledge and legitimacy, or of the potential for dialogue within a liberal consensus in which scientific claims to truth about the social and natural worlds have little place, postmodernists display the same underlying tendency: that is, that there are no universal standards against which science may lay claim in order to validate its standards.

\section{Researches in Problem Solving and Decision Making}

Researchers always plunk firm on accepting the challenges related to the complexities of diverse sectors via the active use of diverse research methods. Nevertheless, the vivacious use of research methods requires acquaintance with research knowledge and diverse methodologies. Usually researches are funded by different agencies including organizations, agencies, companies, institutions etc. As a practice-based activity research can take a concrete shape when creative works are considered both the research and the object of research itself. Focusing on the required information through the methodology of the project, discussion and results, research becomes a movement from known to unknown and even unknown to known (simple at the beginning and complex at last at findings), a voyage of discovery of some sort which will be new of its kind. Discovering of truth with the help of study, observation, comparison and experiment, research hence transpire to be the art of investigative research. The usefulness of researches in everyday life is such that it turns out to be a methodical nevertheless logical gaze for appropriate information of a specific topic directed to search knowledge and truth in every day life. As a matter of fact researches in every day life are embedded with problem solving and decision making activities for all and everyone------may be a common man or elite, a manager, a layman etc. Sekaran (1992) has identified the benefits of research knowledge to managers of ventures as follows:

- Identify and solve small problems in the work setting.

- Know how to discriminate good from bad research.

- Appreciate and constantly remember the multiple influences and multiple effects of factors impinging on a situation.

- Take calculated risks in decision making, knowing full well the probabilities attached to the different/possible outcomes.

- Prevent possible vested interests from operating in a situation.

- Relate to hired researchers and consultants more effectively.

Everyday life is unusual always in different places. For example it would be interesting to study the workers as guinea pigs who have probably been infected with virus of everyday life because they, having no access to specialized activities, have no life except everyday life. The manner of investigating the common people in search of an unusual uniqueness of everyday life clearly reveals a desire to hide behind a development of thought based on the separation of artificial, fragmentary area so as to reject the ineffective, rude and worrying concept of everyday life.

Everyday life is not the whole thing ---even though its overlapping with specialized activities is such that in a sense researches and researchers are never outside of everyday life. Issues 
of everyday life are always a hot cake issues for research study and have to place everyday life at the center of everything. It ought to be remembered that every task (research project) begins from it and every achievement returns to it to get its real significance. Everyday life is the measure of all things: of the fulfillment, non-fulfillment of human relations; of the use of lived time; of creative testing; and of radical politics. What build for the complexity of distinguishing an environment of everyday life is not only the reality that it has already become the perceived meeting ground of an empirical sociology/anthropology or any discipline and a conceptual explanation, but also the reality that it turn out to be the stake or venture in any radical revitalization of culture, society and other issues.

\section{Conclusion}

In our everyday life we inquire and wonder on diverse issues and matters. Using our sense and inquisitive knowledge background we explore the reasons behind any issue. Researches and everyday life are embedded and researches can be accomplished in everyday life situation. It means researches are also very much embedded with everyday life of the commoners. There is nothing ordinary about the ordinary. That is, if by ordinary we mean usual or normal nevertheless, members of every culture believe that their way of doing things is normal. In a research we search the reasons behind the issues. Researches are and can be performed in diverse sectors related especially with issues and problems which are embedded with our routine daily verve, cognitive, social-cultural life and involvements etc.

The fields of science are usually organized along two major lines, Natural sciences dealing with the study of the natural world, and Social sciences with the systematic study of human behavior and society. Social sciences are based on rigorous scientific research techniques focusing on diverse aspects of human and social problems. Human society is a multifarious consign for human and other creatures hence problems are also multifaceted in complex societies which call for methodical, intelligent researching about their causes and also for finding out their solutions. As problems are related to human beings who are both unpredictable and undependable in behavior, consequently diverse methods are prerequisite for understanding and solving the problems and finding out the truth. To resolve diverse problems related to society and to other issues and to satisfy the thirst of knowledge, research is prerequisite and for conducting a good social research we need to pursue a balanced research method.

In the pursuit of knowledge embed with human lives, society and other quarters, social research is principally a unique process of movement, the search for knowledge through objective and systematic method of finding solution to a social problem. Research methods are a fundamental part of the social sciences providing a means through which intellectual development and progress is enhanced. Indeed, the status as 'sciences' is often justified by attaching to the technical features of research methods, while the very term 'science' carries with it ideas of areas of study which are easy to get to only to those who have undergone an wide-ranging training process in order to understand their internal workings. Theory, methods and methodology are all part of the issues and processes that surround and inform the discipline. These differences, however, constantly lead to disputes, as well as confusions, over the nature of research and the methods employed in following its aims and the use in daily life. 
There are diverse schools of thought embedded with key perspectives in social research. Positivism is based on scientific notion with the idea that social science ought to reflect the aims and methods of natural science however the assessment of different methods clarifies that social researches seems less objective compared to natural sciences. Realism's perception is that knowledge the people have of their social world influence their behavior. Feminist are critical of the foundations and aims of science as being male-centered and hierarchical, finishing on a critique of research practice as ethnocentric and racist reveal the diversities in the schools of thought in social research. Postmodernism is often described as an orientation within the philosophy of science, which denies the possibility of acquiring factual knowledge about the world. It believes that what we know about society is our own construction, which we must deconstruct. The great narratives (development, science, freedom, romanticism, truth) are heroic myths that give legitimacy to the existing social order. All these perspectives do not simply dictate the nature of research itself, or how it is hold out; even though the issues will inform how the aims, methods and process of social research are measured.

Focusing on diverse social and cultural ills (problems) a narrow attitude to social research practice supports the idea that theory, ethics, values and methods of social research are distinct topics and those researchers, despite living and participating in the societies that they study, are somehow distinct from the social world which is the object of their investigations. Nevertheless, the findings of their studies may be extremely helpful to the society and for the people living there. In having an understanding of debates and the applicability of different methods of research and the implications of research findings on society, improved research and more inquiring and confident researchers will be more productive. Issues and methods cannot be simply separated and hence researchers will produce more systematic understandings of the social world by being aware of problems, their implications for research and applications of research findings on society and day to day life.

The expediency of researches in everyday life is such that it turns out to be a methodical nevertheless logical gaze for appropriate information of a specific topic directed to search knowledge and truth in every day life. Researches in every day life are embedded with problem solving and decision making activities for all and everyone---may be a common man or elite, a manager, a layman etc.

Everyday life is not the whole thing ---even though its overlapping with specialized activities is such that in a sense researches and researchers are never outside of everyday life. Issues of everyday life are always a hot cake issues for research study and have to place everyday life at the center of everything. Everyday life is the measure of all things: of the fulfillment, non-fulfillment of human relations; of the use of lived time; of creative testing; and of radical politics. What build for the complexity of distinguishing an environment of everyday life is not only the reality that it has already become the perceived meeting ground of an empirical discipline and a conceptual explanation, but also the reality that it turn out to be the stake or venture in any radical revitalization of culture, society and other issues. 


\section{BIBLOGRAPHY}

Bauman L. J (1997). The use of ethnographic interviewing to inform questionnaire construction. Health Educ Q, 19(1), 9-23.

Bourdieu, P., \& Loïc J. D., Wacquan (1992). An invitation to reflexive sociology. Oxford: Polity Press.

Douglas, M. (1966). Purity and danger. Harcourt and Bavourd.

Giddens, A. (1974). “Introduction" in (self ed.). Ppositivism in Sociology. London: Heinemann.

Joshi, P. R. (2002). The scientific research process. Kathmandu Press.

Kerlinger, F. N. (1986). Foundations of behavioral research ( ${ }^{\text {rd }}$ ed.). New York: Holt, Rinehart and Winston.

Kothari, C. R. (2002). Research methodology: Methods and techniques. ( $2^{\text {nd }}$ ed.) New Delhi: Bishwa Prakashan.

Lapin, L. L. (1987). Statistics for modern business decisions. Harcourt Brace Jovanovich, Inc.

Levi Strauss, C. (1964). The raw and the cooked. Weighman, John and Weighman, Doreen, trans. New York: Harper Torch books.

Pandey, T. R. (2008). The field of sociological study: Theories, meta theories and the status of their use in the case of studies in Nepal. In Sociology and Anthropology in Nepal, Proceedings of National Workshop on Past, Present and Future of Sociology/ Anthropology in Nepal. Tribhuvan University, Prithvi Narayan Campus, Pokhara.

Sekaran, U. (1992). Research method in business: A skill building approach. Singapore: John Wiley and Sons.

Upadhyay, P. (2011). Research methods in sociology and anthropology. Kathmandu, Kshitiz Publication.

Young, P. V. (1982). Scientific social survey and research. New Delhi; Prentice Hall of India, Pvt. Limited. 